\title{
All-Optical Two-Color Terahertz Emission from Quasi-2D Nonlinear Surfaces
}

\author{
J. S. Totero Gongora $\odot,{ }^{1, *}$ L. Peters $\odot,{ }^{1}$ J. Tunesi $\odot,{ }^{1}$ V. Cecconi $\odot,{ }^{1}$ M. Clerici $\odot,{ }^{2}$ A. Pasquazi $\odot,{ }^{1}$ and M. Peccianti $\odot^{1, \dagger}$ \\ ${ }^{1}$ Emergent Photonics Lab (EPic), Department of Physics and Astronomy, University of Sussex, Brighton, BN1 9QH, United Kingdom \\ ${ }^{2}$ Ultrafast Nonlinear Optics Lab (UNO), James Watt School of Engineering, University of Glasgow, Glasgow, G12 8QQ, United Kingdom
}

(Received 2 September 2020; revised 22 October 2020; accepted 18 November 2020; published 21 December 2020)

\begin{abstract}
Two-color terahertz (THz) generation is a field-matter process combining an optical pulse and its second harmonic. Its application in condensed matter is challenged by the lack of phase matching among multiple interacting fields. Here, we demonstrate phase-matching-free two-color $\mathrm{THz}$ conversion in condensed matter by introducing a highly resonant absorptive system. The generation is driven by a third-order nonlinear interaction localized at the surface of a narrow-band-gap semiconductor, and depends directly on the relative phase between the two colors. We show how to isolate the third-order effect among other competitive THz-emitting surface mechanisms, exposing the general features of the two-color process.
\end{abstract}

DOI: 10.1103/PhysRevLett.125.263901

The nonlinear generation of broadband terahertz $(\mathrm{THz})$ fields from ultrafast optical pulses is a subject of great interest for fundamental research and disruptive applications in imaging, spectroscopy, and the design of materials and devices [1-12]. Current research aims to identify new materials and generation mechanisms to boost the efficiency and versatility of nonlinear $\mathrm{THz}$ sources, as in the case of organic crystals [13,14], spintronic substrates [15], and tunable gas lasers [16]. Second-order optical rectification (OR) in $\chi^{(2)}$ bulk crystals, such as $\mathrm{ZnTe}$ or $\mathrm{LiNbO}_{3}[17,18]$ has been at the core of bright $\mathrm{THz}$ source development for many years. The stringent requirement to maintain the longitudinal phase-matching condition across a wide optical bandwidth, however, strongly limits both the choice of laser sources and nonlinear materials. While phase matching can be achieved via sophisticated settings, it certainly poses growing challenges when synchronous propagation of multiple fields is required, as in the case of third-order phenomena. To overcome the limitations imposed by phase matching, a promising alternative is provided by new types of emitters capable of high-conversion efficiency over short propagation distances [13], as in the case of ultrathin spintronic substrates $[15,19,20]$. In this context, narrowband-gap semiconductor surfaces have emerged as remarkably efficient surface $\mathrm{THz}$ sources, under ultrafast illumination (typically 100 fs class pulses). Indium arsenide (InAs), in particular, provides exceptionally high conversion efficiencies per unit length and stands as one of the standard benchmarks for surface nonlinear $\mathrm{THz}$ generation [21].

Published by the American Physical Society under the terms of the Creative Commons Attribution 4.0 International license. Further distribution of this work must maintain attribution to the author(s) and the published article's title, journal citation, and DOI.
While at lower fluences $\left(<100 \mathrm{~nJ} / \mathrm{cm}^{2}\right)$, InAs sources are mostly driven by carrier diffusion dynamics [22], in the high-pump regime $\left(>10 \mu \mathrm{J} / \mathrm{cm}^{2}\right)$ their emission is dominated by dc-biased surface optical rectification (DC-SOR) [23-25]. Similarly to second-harmonic generation from centrosymmetric surfaces [26], in DC-SOR the optical pump interacts with a symmetry-broken medium provided by the surface depletion field $E_{\text {surf }}$ of the semiconductor, which leads to an effective quadratic response in the proximity of the surface [27]. Because of the neutralization of the surface field from excited photocarriers the emission from DC-SOR typically saturates at high pumping fluences [21,28]. This saturation, however, does not affect directly the third-order $\chi^{(3)}$ nonlinearity, which can be used to generate $\mathrm{THz}$ radiation by mixing an optical pulse with its second-harmonic through a process known as two-color optical rectification:

$$
E_{\mathrm{THz}}(t) \propto \chi^{(3)}(\Omega, 2 \omega,-\omega,-\omega) E_{2 \omega}(t) E_{\omega}^{*}(t) E_{\omega}^{*}(t),
$$

where $E_{\omega}(t)$ and $E_{2 \omega}(t)$ are the electric fields of the optical and second-harmonic pulses, and ${ }^{*}$ denotes the complex conjugate. The key signature of this interaction is the dependence of the $\mathrm{THz}$ amplitude from the mutual phase and frequencies of the interacting fields $[29,30]$, a degree of control generally not attainable in $\chi^{(2)}$-based $\mathrm{THz}$ sources. Two-color generation has been extensively exploited in laser-induced gas plasmas, leading to the demonstration of THz sources with record-breaking bandwidths [31-38]. In these systems, the role of the four-wave-mixing is negligible, as demonstrated by Ref. [39], whereas the THz emission is rather the result of highly nonperturbative mechanisms. Two-color excitation also plays a key role in the injection and coherent control of ultrafast ballistic currents in bulk semiconductors [40-43]. In these experiments, the interference between two quantum pathways, single-photon 
absorption at $2 \omega$ and two-photon absorption at $\omega$, leads to the ultrafast injection of directional currents acting as $\mathrm{THz}$ sources $[41,44,45]$. Quantum interference is mediated by a below-band-gap optical illumination seeding the two-photon absorption process $\left(\hbar \omega<E_{g}<2 \hbar \omega\right.$, where $E_{g}$ is the semiconductor band gap). However, in the presence of highly above-band-gap illumination in narrow-band-gap semiconductors, $\left(E_{g}<\hbar \omega\right)$, the direct one-photon absorption is the dominant mechanism, effectively reducing the number of photons available for multiphoton nonlinear current injection processes. [45]. A central challenge in implementing Eq. (1) is in keeping synchronous interactions among all the interacting fields, a condition practically out of reach in bulk condensed matter.

In this Letter, we demonstrate, for the first time, two-color optical rectification at an air-InAs interface via a highly resonant above-band-gap excitation, a process we denote as all-optical surface optical rectification (AO-SOR) to distinguish it from the DC-SOR. The strong absorbing regime is responsible for the very high effective $\chi^{(3)}$ nonlinearity and results in a deeply subwavelength penetration depth that localizes the interaction within a quasi-2D surface (in the scale of 25 atomic layers). The dimensionally reduced interaction length allows the relaxation of typical phasematching constraints found in bulks. It also affects the dynamics of competitive surface mechanisms, e.g., the photo-carrier-driven screening, on the emission. Figure 1 illustrates our experimental setup comprising a fundamental $(\mathrm{FH})$ excitation beam $(\lambda=800 \mathrm{~nm}, 100 \mathrm{fs}, 1 \mathrm{kHz}, \approx 1.0 \mathrm{~mJ}$, red beam) copropagating with its second-harmonic (SH) signal $(\lambda=400 \mathrm{~nm}, \approx 35.4 \mu \mathrm{J}$, blue beam). The crosspolarized SH pulse at $400 \mathrm{~nm}$ was generated via type-I process (oo-e) in a $0.1 \mathrm{~mm}$ thick $\beta$-barium borate (BBO) crystal. A birefringent calcite plate $(C P)$ [46] introduced a tunable phase delay $\tau_{\mathrm{CP}}(\phi)$ between the two cross-polarized fields. Specifically, the phase delay is due to the difference in optical path length between the ordinary wave at $\omega$ and the extraordinary wave at $2 \omega$ [47]. A second half-wave plate at $800 \mathrm{~nm}$ rotated the polarization of the fundamental field, resulting in the two pumps being copolarized. As a nonlinear surface, we exploited an undoped $\langle 100\rangle$ InAs substrate. The pumps illuminated the surface at $45^{\circ}$ with respect to its normal. The $\mathrm{THz}$ signal was collected along the specular reflection direction (green beam) through a standard timedomain spectroscopy (TDS) setup, implemented via a nonlinear $\langle 110\rangle \mathrm{ZnTe}$ crystal [48]. In this configuration, the generated $\mathrm{THz}$ field consists of three major contributions:

$$
\begin{aligned}
E_{\mathrm{THz}}(t) \propto & \chi^{(3)} E_{\omega}(t) E_{\omega}^{*}(t) E_{\mathrm{surf}} \\
& +\chi^{(3)} E_{2 \omega}\left(t-\tau_{\mathrm{CP}}\right) E_{2 \omega}^{*}\left(t-\tau_{\mathrm{CP}}\right) E_{\mathrm{surf}} \\
& +\chi^{(3)} E_{2 \omega}\left(t-\tau_{\mathrm{CP}}\right) E_{\omega}^{*}(t) E_{\omega}^{*}(t) \cos \left(2 \omega \tau_{\mathrm{CP}}\right) .
\end{aligned}
$$

The first two terms account for separate DC-SOR contributions from the fundamental $E_{\omega}$ and second-harmonic $E_{2 \omega}$
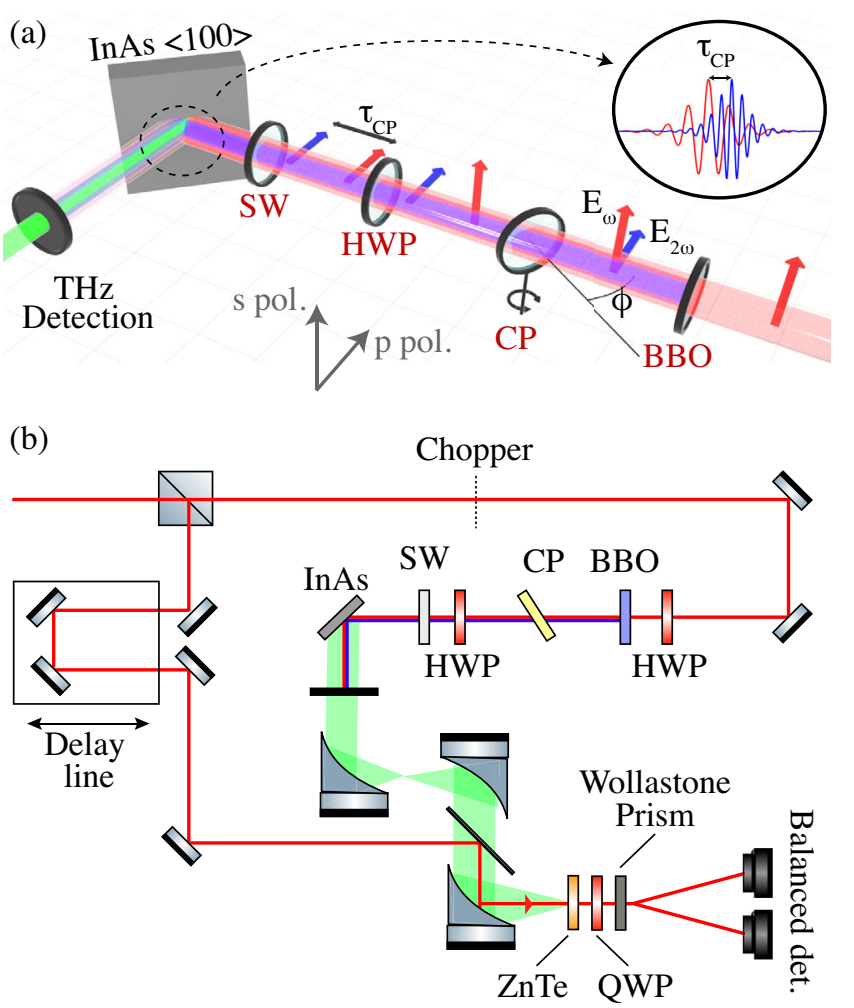

FIG. 1. Experimental setup. (a) The red, blue, and green beams denote the $800,400 \mathrm{~nm}$, and $\mathrm{THz}$ beam paths, respectively. The inset displays the role played by the phase delay. (b) A schematic of the whole setup. Half-wave plate (HWP), barium borate crystal (BBO), Calcite plate (CP), fused silica window (SW: UV), Quarter-wave plate (QWP), zinc telluride ( $\mathrm{ZnTe}$ ).

fields [Fig. 2(a)]. The third term, conversely, represents the AO-SOR mechanism under investigation, where fundamental and SH fields interact through the third-order $\chi^{(3)}$ process from Eq. (1) [Fig. 2(b)], where $\tau_{\mathrm{CP}}$ is the delay between $E_{\omega}$ (a)
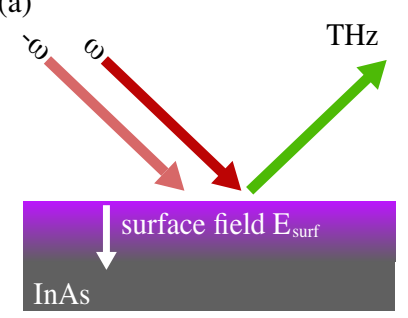

(c)

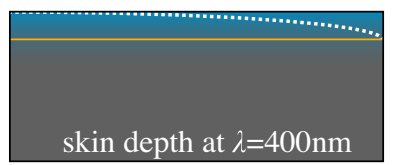

(b)
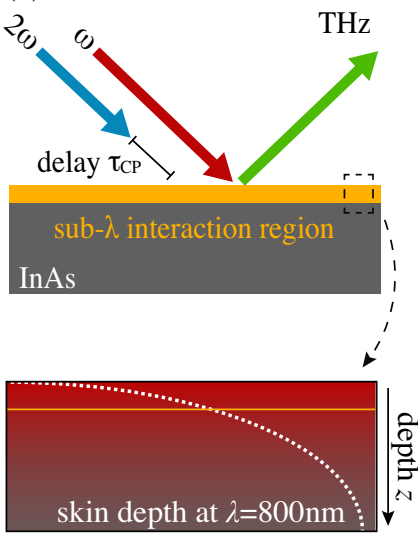

FIG. 2. THz emission from (a) DC-SOR and (b) AO-SOR. In (c), the interaction region (yellow line) is compared to the skin depth of the interacting fields, $\lambda=400$ and $\lambda=800 \mathrm{~nm}$, respectively. 


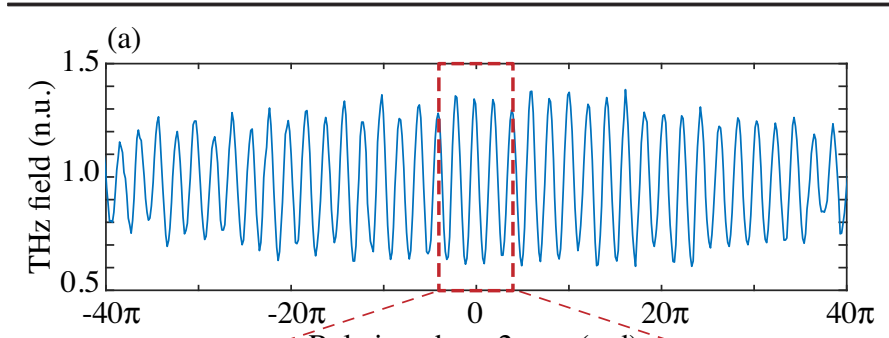

(c)

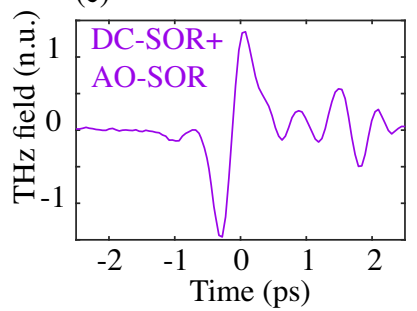

(b)
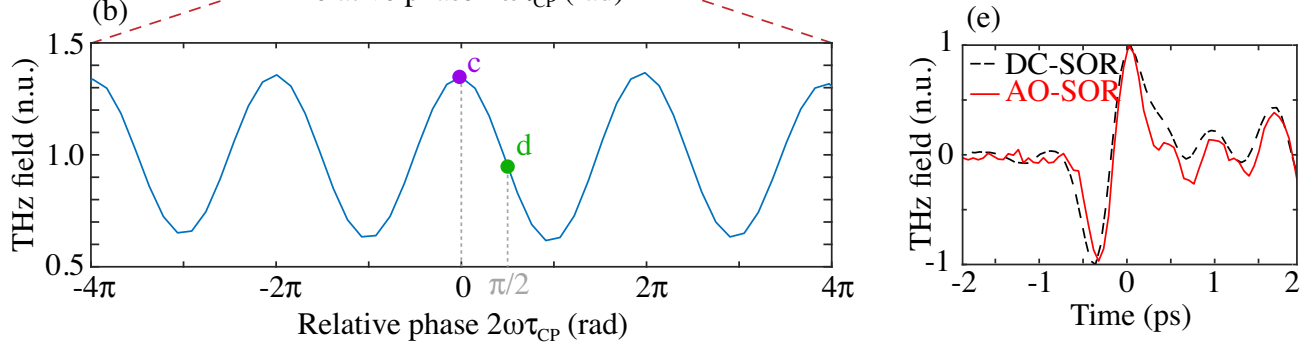

(d)

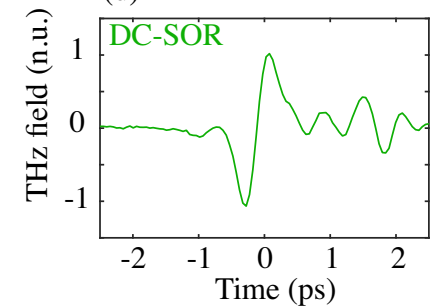

(f)

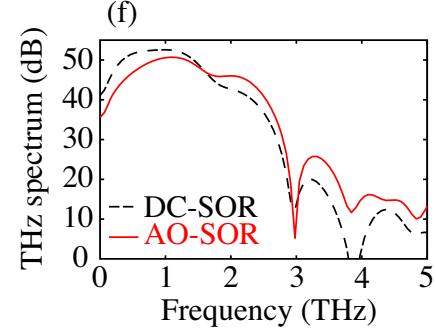

FIG. 3. (a),(b) Peak THz emitted field as a function of the phase delay $\tau_{\mathrm{CP}}$. In both plots, the THz field has been normalized to the DC-SOR contribution. (c),(d) Detected THz pulse for $\tau_{\mathrm{CP}}=0$ and $\tau_{\mathrm{CP}}=\pi / 4 \omega$, respectively. In both cases, we considered $p$-polarized excitation and detection. (e),(f) Comparison of the pulse temporal and spectral amplitudes obtained by AO-SOR (red line) and DC-SOR (black dashed line), respectively. In these experiments, the incident pump energy was $1.2 \mathrm{~mJ}$.

and $E_{2 \omega}$. We neglected any term arising from carrier dynamics as they are highly saturated at the considered fluences $[21,28]$. Both DC-SOR and AO-SOR are localized within the subwavelength region defined by the short skin depth of the incident optical fields (approximately $140 \mathrm{~nm}$ at $\lambda_{\omega}=800$ and $16 \mathrm{~nm}$ at $\lambda_{2 \omega}=400 \mathrm{~nm}$ ) [49]. In this condition, longitudinal phase matching does not play a significant role in the emission [Fig. 2(c)]. The delay $\tau_{\mathrm{CP}}$ is controlled by rotating the calcite plate [47], and it affects only the amplitude of the all-optical process. By holding the TDS delay in the position of maximum field detected, a change of $\tau_{\mathrm{CP}}$ produces a beating pattern of period $1 /(2 f)=$ $\pi / \omega$ [Figs. 3(a)-3(b)], as predicted by Eq. (2). When $\tau_{\mathrm{CP}}=0$, all the sources components are in phase, resulting in a peak in the emission. Interestingly, in this case, the emitted $\mathrm{THz}$ field is enhanced by a factor of $\approx 40 \%$ (96\% in power) [Fig. 3(c)] when considering an incident pump energy of $1.2 \mathrm{~mJ}$. When $\tau_{\mathrm{CP}}=\pi / 4 \omega$, conversely, the AO-SOR component is suppressed, and we can isolate the DC-SOR contribution [Fig. 3(d)]. By subtracting the two signals, we can thus determine the $\mathrm{THz}$ field generated solely by AO-SOR. As illustrated in Figs. 3(e), 3(f), the THz pulse generated by AO-SOR is shorter in time and broader in spectrum than that from DC-SOR. In the range $2-2.5 \mathrm{THz}$ the AO-SOR power spectral density is $3.5-4 \mathrm{~dB}$ above the corresponding spectrum for the DC-SOR. It is worth noting that our current ZnTe-based detection limits the appreciable bandwidth creating a slope in the spectrum as the frequency approaches $3 \mathrm{THz}$. This limitation, however, could be removed by employing different nonlinear detection crystals operating at different wavelengths, such as GaP [50], or an air-ABCD detection scheme [51]. We obtained further insights into the AO-SOR emission mechanism by measuring the emitted $\mathrm{THz}$ field as a function of the incident optical power, as summarized in Fig. 4. To this end, we measured the peak $\mathrm{THz}$ field as a function of $\tau_{\mathrm{CP}}$ and the incident pump energy [Fig. 4(a)]. As shown in Fig. 4(b), for each value of the pumping power we can employ the interference signal (green line) to isolate the DC-SOR contribution (mean value of the oscillation, dashed blue line) from the cosinelike AO-SOR contribution (amplitude of the interference oscillations, dashed orange line. For each pump energy, we extracted the individual contributions by fitting the experimental data (red circles). The analysis of these contributions is particularly useful to discriminate the saturation dynamics of the two competing mechanisms, as illustrated in Fig. 3(c). The DC-SOR contribution (blue dots) shows an initial linear trend at lower energies, followed by a soft saturation profile characteristic of the field-induced DC-SOR $[21,28]$. The AO-SOR, conversely, does not show any appreciable saturation effect at the high fluences of our experiment, as the mechanism is not affected by the screening of the semiconductor surface field. Note that direct $\mathrm{THz}$ photocarriers screening tends to be also negligible due to the extremely short skin depth of the second harmonic that defines the interaction region. This result is illustrated in Fig. 4(d): by extracting the AO-SOR field contribution, we observe how it increases quadratically with the square of the input optical energy $\left[P_{\text {pump }}^{2} \propto\left|E_{\omega}\right|^{4}\right.$, Fig. 4(d)] as dictated by Eq. (1) in the presence of a second-harmonic field of the form $E_{2 \omega} \propto E_{\omega}^{2}$. Quite interestingly, we were able to observe the onset of a subquartic trend for high pump energies. The main contributors to this effect are potentially the self-phase and cross-phase modulation in the BBO crystal, which can induce a change of the nonlinear product at the InAs surface by slightly detuning the fundamental and $\mathrm{SH}$ waves at high 

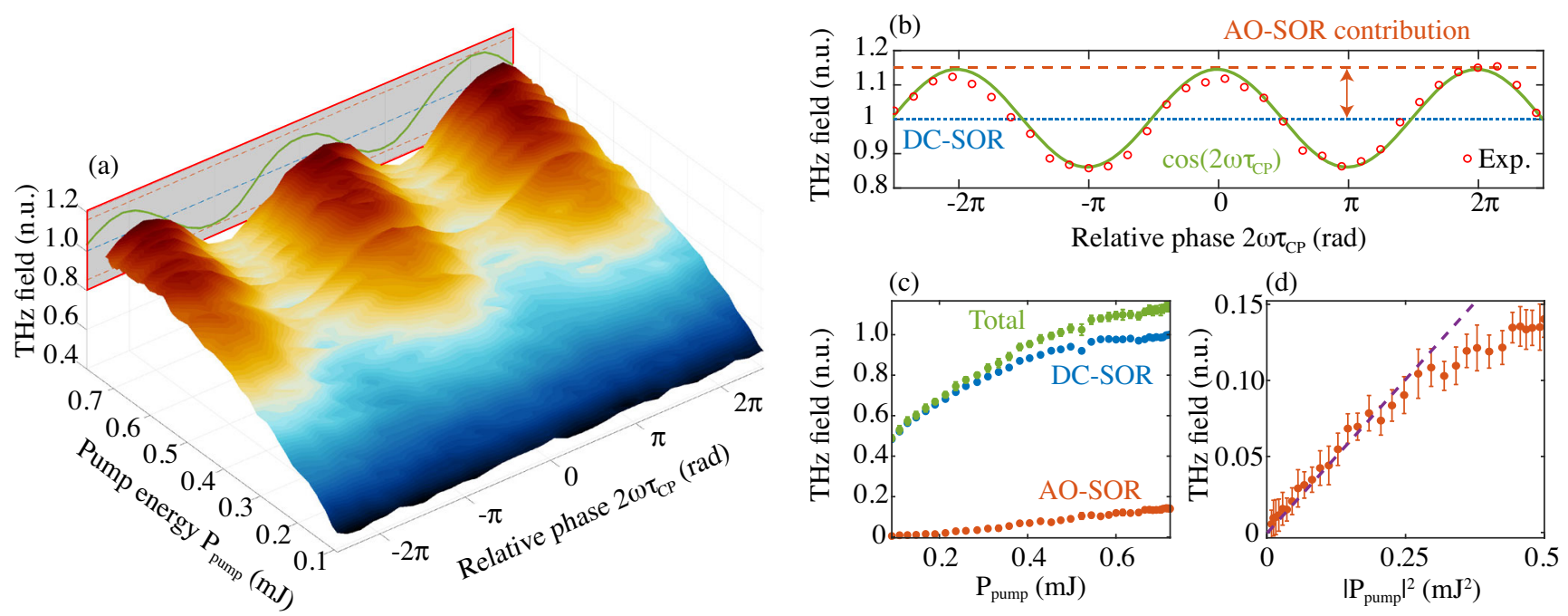

FIG. 4. (a),(b) THz peak emission as a function of the phase delay $\tau_{\mathrm{CP}}$ and the pump energy $P_{\omega}$. For each pump energy, we extracted the SOR contribution [panel (b), blue line], corresponding to the center value, and the AO-SOR contribution (orange line), corresponding to the beating amplitude. For each pump value, we extracted these values by fitting the experimental data (red circles). (c) Total emission and DC-SOR contribution as a function of the pump power, highlighting the soft saturation at high pump energies characteristic of the DC-SOR emission mechanism. (d) AO-SOR emission as a function of $\left|P_{\text {pump }}\right|^{2}$. The dashed line highlights the fitted ideal quartic dependence.

pump rates. Further insights on the AO-SOR process can be obtained by observing the $\mathrm{THz}$ emission dependence from crystal rotation. To this end, we analytically calculated such a dependence by generalizing the full-vector approach described in Refs. [52-54]. We derived a set of nonlinear Fresnel reflection coefficients for the $p$ and $s$ polarization components by matching the incident fields with the nonlinear polarization field $P_{\mathrm{NL}}$ generating the $\mathrm{THz}$ field. Following the standard approach outlined in Ref. [55], we obtained the $\mathrm{THz}$ field generated in reflection by matching $P_{\mathrm{NL}}$ with a wave reflected at the air-InAs interface. Our calculations show that the crystal orientation affects each generation mechanism differently. A particularly relevant case involves the $s$-polarized THz field generated by a $\langle 100\rangle$ InAs by $p$-polarized pumps. In this configuration, the standard DC-SOR is suppressed by the symmetry properties of the nonlinear tensor $\chi^{(3)}$ [23,28]. The two-color $\mathrm{THz}$ emission, on the contrary, reads as follows:

$$
\begin{aligned}
E_{p, s}= & \frac{3}{4} \Omega L_{\mathrm{eff}} A_{s} f_{c}^{2} f_{2 c} t_{p}^{2} t_{2 p} \\
& \sin (4 \phi)\left[\chi_{i i i i}-2 \chi_{i i j j}-\chi_{i j j i}\right] E_{2 \omega} E_{\omega}^{2},
\end{aligned}
$$

where $\phi$ is the crystal orientation angle measured from the 100 axis, and $\chi_{i i i i}, \chi_{i i j j}$, and $\chi_{i j j i}$ are the three independent components of the tensor $\chi^{(3)}$. To ease a direct comparison with the DC-SOR case, all the other quantities in Eq. (3) are identical to those in Ref. [28]. Our experiments captured the fourfold symmetry predicted by Eq. (3), as illustrated in Fig. 5. These results confirm that the AO-SOR emission originates from the nonlinear susceptibility tensor of InAs. Interestingly, Eq. (3) allows us to also estimate the value of the nonlinear susceptibility $\chi^{(3)}$ for the AO-SOR process in InAs as $\chi^{(3)} \approx 9 \times 10^{-21} \mathrm{~m}^{2} / \mathrm{V}^{2}$.

In conclusion, we provide here the first experimental demonstration of phase-matching free, two-color $\mathrm{THz}$ generation in condensed matter. This process, which is not observed in bulk crystals, occurs in highly absorptive systems within a sub-wavelength interaction region dictated

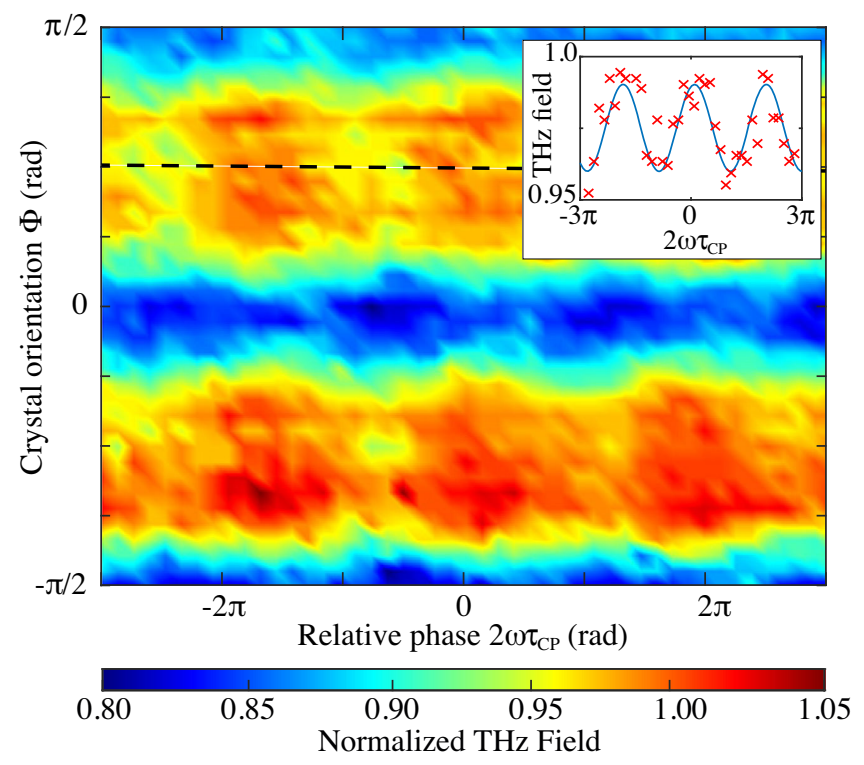

FIG. 5. AO-SOR THz emission as a function of the crystal orientation angle $(\varphi)$ and the mutual delay $\tau_{\mathrm{CP}}$. As predicted by analytical theory, the emission is characterized by a fourfold symmetry which descends from the symmetry properties of the nonlinear susceptibility $\chi^{(3)}$ of InAs. (inset) Peak-field trace as a function of $2 \omega \tau_{\mathrm{CP}}$ for $\phi=\pi / 4$ (dashed line). 
by the skin depth of the SH field ( $16 \mathrm{~nm}$ at $\lambda=400 \mathrm{~nm})$. The reduced interaction length acts as a quasi-2D structure and ensures that the optical-to-THz conversion is unaffected by the longitudinal phase mismatch between the interacting waves. We predict that this approach could be ideal for the generation of $\mathrm{THz}$ waves with large frequency detuning between the two-color excitations, opening to the realization of non-zero-frequency-carrier $\mathrm{THz}$ parametric amplification. Our results also suggest that AO-SOR can significantly outperform DC-SOR for the same total input pump power. Indeed, in our experiments, a minor amount of SH contribution (about $3.5 \%$ in Fig. 3) produces a macroscopic enhancement of the optical to $\mathrm{THz}$ conversion from InAs. If we denote as $\alpha$ the total fractional power of the secondharmonic generated from the $800 \mathrm{~nm}$ pump, the THz fields increase as $E_{\mathrm{THz}} \propto(1-\alpha) \sqrt{\alpha}$. Such a function has a very steep gradient around $\alpha \approx 3.5 \%$ and peaks at about $\alpha \approx 33 \%$. Although this consideration does not account for the losses, it indicates that this process yields a much higher net efficiency when compared to standard benchmark surface emitters. We believe that our proof-of-concept results could seed new developments in the implementation of ultrathin $\mathrm{THz}$ emitters for, e.g., near field imaging applications and integrated nanophotonic devices, in which traditional nonlinear crystals cannot be scaled to the same size without reducing to unpractical levels the optical- $\mathrm{THz}$ conversion efficiency $[11,12,56]$. Besides, the lack of any observable physical saturation mechanisms typically related to photocarrier mediated screening of the surface field in DC-SOR, strongly suggests that the conversion limit of AO-SOR is set by mechanisms that become relevant only close to the damage threshold of the substrate.

The datasets for all figures are freely accessible at [57].

J. S. T. G. acknowledges funding from the University of Sussex (Helena Normanton Fellowship) and the Leverhulme Trust (Leverhulme Early Career Fellowship ECF-2020-537). M. C. acknowledges the support from the UK Research and Innovation (UKRI) and the UK Engineering and Physical Sciences Research Council (EPSRC) (Innovation Fellowship Programme, Grant No. EP/S001573/1). A. P. acknowledges the support from the UK Research and Innovation (UKRI) and the UK Engineering and Physical Sciences Research Council (EPSRC) (Innovation Fellowship Programme, Grant No. EP/S001018/1). This project received funding from the European Research Council (ERC) under the European Union's Horizon 2020 Research and Innovation Programme (Grant No. 725046 "TIMING" and Grant No. 851758 “TELSCOMBE”).

*j.totero-gongora@sussex.ac.uk

m.peccianti@sussex.ac.uk

[1] C. G. Wade, N. ibalic, N. R. de Melo, J. M. Kondo, C. S. Adams, and K. J. Weatherill, Nat. Photonics 11, 40 (2017).
[2] U. Fruhling, M. Wieland, M. Gensch, T. Gebert, B. Schutte, M. Krikunova, R. Kalms, F. Budzyn, O. Grimm, J. Rossbach, E. Plnjes, and M. Drescher, Nat. Photonics 3, 523 (2009).

[3] T. Kampfrath, K. Tanaka, and K. A. Nelson, Nat. Photonics 7, 680 (2013).

[4] C. Kealhofer, W. Schneider, D. Ehberger, A. Ryabov, F. Krausz, and P. Baum, Science 352, 429 (2016).

[5] S. Baierl, M. Hohenleutner, T. Kampfrath, A. K. Zvezdin, A. V. Kimel, R. Huber, and R. V. Mikhaylovskiy, Nat. Photonics 10, 715 (2016).

[6] V. Jelic, K. Iwaszczuk, P. H. Nguyen, C. Rathje, G. J. Hornig, H. M. Sharum, J. R. Hoffman, M. R. Freeman, and F. A. Hegmann, Nat. Phys. 13, 591 (2017).

[7] E. A. Nanni, W. R. Huang, K.-H. Hong, K. Ravi, A. Fallahi, G. Moriena, R. J. Dwayne Miller, and F. X. Krtner, Nat. Commun. 6, 8486 (2015).

[8] D. A. Walsh, D. S. Lake, E. W. Snedden, M. J. Cliffe, D. M. Graham, and S. P. Jamison, Nat. Commun. 8, 1 (2017).

[9] K. Sengupta, T. Nagatsuma, and D. M. Mittleman, National electronics review 1, 622 (2018).

[10] J. Zhang, X. Chen, S. Mills, T. Ciavatti, Z. Yao, R. Mescall, H. Hu, V. Semenenko, Z. Fei, H. Li, V. Perebeinos, H. Tao, Q. Dai, X. Du, and M. Liu, ACS Photonics 5, 2645 (2018).

[11] L. Olivieri, J. S. T. Gongora, A. Pasquazi, and M. Peccianti, ACS Photonics 5, 3379 (2018).

[12] L. Olivieri, J. S. T. Gongora, L. Peters, V. Cecconi, A. Cutrona, J. Tunesi, R. Tucker, A. Pasquazi, and M. Peccianti, Optica 7, 186 (2020).

[13] C. Vicario, A. V. Ovchinnikov, S. I. Ashitkov, M. B. Agranat, V. E. Fortov, and C. P. Hauri, Opt. Lett. 39, 6632 (2014).

[14] C. Vicario, B. Monoszlai, and C. P. Hauri, Phys. Rev. Lett. 112, 213901 (2014).

[15] T. Seifert et al., Nat. Photonics 10, 483 (2016).

[16] P. Chevalier, A. Amirzhan, F. Wang, M. Piccardo, S. G. Johnson, F. Capasso, and H. O. Everitt, Science 366, 856 (2019).

[17] a. Rice, Y. Jin, X. F. Ma, X. C. Zhang, D. Bliss, J. Larkin, and M. Alexander, Appl. Phys. Lett. 64, 1324 (1994).

[18] H. Hirori, A. Doi, F. Blanchard, and K. Tanaka, Appl. Phys. Lett. 98, 091106 (2011).

[19] Y. Wu, M. Elyasi, X. Qiu, M. Chen, Y. Liu, L. Ke, and H. Yang, Adv. Mater. 29, 1603031 (2017).

[20] M. Chen, R. Mishra, Y. Wu, K. Lee, and H. Yang, Adv. Opt. Mater. 6, 1800430 (2018).

[21] L. Peters, J. Tunesi, A. Pasquazi, and M. Peccianti, Nano Energy 46, 128 (2018).

[22] V. Apostolopoulos and M. Barnes, J. Phys. D 47, 374002 (2014).

[23] M. Reid, I. V. Cravetchi, and R. Fedosejevs, Phys. Rev. B 72, 035201 (2005).

[24] L. Peters, J. Tunesi, A. Pasquazi, and M. Peccianti, Sci. Rep. 7, 9805 (2017).

[25] A. Tomasino, A. Mazhorova, M. Clerici, M. Peccianti, S.-P. Ho, Y. Jestin, A. Pasquazi, A. Markov, X. Jin, R. Piccoli, S. Delprat, M. Chaker, A. Busacca, J. Ali, L. Razzari, and R. Morandotti, Optica 4, 1358 (2017).

[26] M. Cazzanelli and J. Schilling, Appl. Phys. Rev. 3, 011104 (2016). 
[27] S. L. Chuang, S. Schmitt-Rink, B. I. Greene, P. N. Saeta, and A. F. J. Levi, Phys. Rev. Lett. 68, 102 (1992).

[28] M. Reid and R. Fedosejevs, Appl. Phys. Lett. 86, 011906 (2005).

[29] Z. Zhang, Y. Chen, S. Cui, F. He, M. Chen, Z. Zhang, J. Yu, L. Chen, Z. Sheng, and J. Zhang, Nat. Photonics 12, 554 (2018).

[30] S. W. Jolly, N. H. Matlis, F. Ahr, V. Leroux, T. Eichner, A.-L. Calendron, H. Ishizuki, T. Taira, F. X. Krtner, and A. R. Maier, Nat. Commun. 10, 2591 (2019).

[31] M. Clerici, M. Peccianti, B. E. Schmidt, L. Caspani, M. Shalaby, M. Giguere, A. Lotti, A. Couairon, F. Legare, T. Ozaki, D. Faccio, and R. Morandotti, Phys. Rev. Lett. 110, 253901 (2013).

[32] N. V. Vvedenskii, A. I. Korytin, V. A. Kostin, A. A. Murzanev, A. A. Silaev, and A. N. Stepanov, Phys. Rev. Lett. 112, 055004 (2014).

[33] P. G. Martinez, I. Babushkin, L. Berge, S. Skupin, E. Cabrera-Granado, C. Kohler, U. Morgner, A. Husakou, and J. Herrmann, Phys. Rev. Lett. 114, 183901 (2015).

[34] V. Andreeva, O. Kosareva, N. Panov, D. Shipilo, P. Solyankin, M. Esaulkov, P. G. de Alaiza Martinez, A. Shkurinov, V. Makarov, L. Berge, and S. Chin, Phys. Rev. Lett. 116, 063902 (2016).

[35] V. A. Kostin, I. D. Laryushin, A. A. Silaev, and N. V. Vvedenskii, Phys. Rev. Lett. 117, 035003 (2016).

[36] Z. Zhang, Y. Chen, M. Chen, Z. Zhang, J. Yu, Z. Sheng, and J. Zhang, Phys. Rev. Lett. 117, 243901 (2016).

[37] L.-L. Zhang, W.-M. Wang, T. Wu, R. Zhang, S.-J. Zhang, C.-L. Zhang, Y. Zhang, Z.-M. Sheng, and X.-C. Zhang, Phys. Rev. Lett. 119, 235001 (2017).

[38] A. D. Koulouklidis, C. Gollner, V. Shumakova, V. Y. Fedorov, A. Pugžlys, A. Baltuška, and S. Tzortzakis, Nat. Commun. 11, 292 (2020).

[39] K. Y. Kim, J. H. Glownia, A. J. Taylor, and G. Rodriguez, Opt. Express 15, 4577 (2007).
[40] E. Dupont, P. B. Corkum, H. C. Liu, M. Buchanan, and Z. R. Wasilewski, Phys. Rev. Lett. 74, 3596 (1995).

[41] D. Cote, J. M. Fraser, M. DeCamp, P. H. Bucksbaum, and H. M. van Driel, Appl. Phys. Lett. 75, 3959 (1999).

[42] J. Gudde, M. Rohleder, T. Meier, S. W. Koch, and U. Hofer, Science 318, 1287 (2007).

[43] S. Sederberg, F. Kong, F. Hufnagel, C. Zhang, E. Karimi, and P. B. Corkum, Nat. Photonics 14, 680 (2020).

[44] J. B. Khurgin, J. Nonlinear Opt. Phys. Mater. 04, 163 (1995).

[45] M. Spasenovic, M. Betz, L. Costa, and H. M. van Driel, Phys. Rev. B 77, 085201 (2008).

[46] EKSMA BBAR@800+400 nm - 310-450 fsec.

[47] K. A. Stankov, V. P. Tzolov, and M. G. Mirkov, Appl. Phys. B 54, 303 (1992).

[48] Q. Wu, M. Litz, and X. C. Zhang, Appl. Phys. Lett. 68, 2924 (1996).

[49] D. E. Aspnes and A. A. Studna, Phys. Rev. B 27, 985 (1983).

[50] Q. Wu and X.-C. Zhang, Appl. Phys. Lett. 70, 1784 (1997).

[51] J. Dai, X. Xie, and X.-C. Zhang, Phys. Rev. Lett. 97, 103903 (2006).

[52] N. Bloembergen and P. S. Pershan, Phys. Rev. 128, 606 (1962).

[53] P. N. Saeta and N. A. Miller, Appl. Phys. Lett. 79, 2704 (2001).

[54] W. Hubner, K. H. Bennemann, and K. Bohmer, Phys. Rev. B 50, 17597 (1994).

[55] R. W. Boyd, Nonlinear Optics, 3rd ed. (Academic Press, Amsterdam, Boston, 2008).

[56] J. Zhao, Y. E, K. Williams, X.-C. Zhang, and R. W. Boyd, Light Sci. Appl. 8, 55 (2019).

[57] https://doi.org/10.6084/m9.figshare.13325468. 